\title{
Le cri du paon dans la gueule du dragon
}

Les autres voix dans les nouvelles de Flannery O'Connor

\section{Claudia Desblaches}

\section{(2) OpenEdition}

\section{Journals}

Édition électronique

URL : https://journals.openedition.org/clo/1904

DOI : $10.4000 /$ clo. 1904

ISSN : 2266-1816

Éditeur

INALCO

\section{Édition imprimée}

Date de publication : 1 janvier 2014

ISBN : 978-2-85831-222-1

ISSN : 0396-891X

Référence électronique

Claudia Desblaches, «Le cri du paon dans la gueule du dragon », Cahiers de littérature orale [En ligne], 75-76 | 2014, mis en ligne le 29 avril 2015, consulté le 02 juillet 2021. URL : http:// journals.openedition.org/clo/1904; DOI : https://doi.org/10.4000/clo.1904

Ce document a été généré automatiquement le 2 juillet 2021

\section{(c) (†) \&}

Cahiers de littérature orale est mis à disposition selon les termes de la Licence Creative Commons Attribution - Pas d'Utilisation Commerciale 4.0 International. 


\title{
Le cri du paon dans la gueule du dragon
}

Les autres voix dans les nouvelles de Flannery O'Connor

\author{
Claudia Desblaches
}

\section{Introduction : crier à l'oreille des sourds et des malentendants}

1 Flannery O'Connor explique dans ses Conversations (Magee, 1952, 113) la nécessité pour l'écrivain de «crier à l'oreille des sourds » et d'aider les personnages en manque de foi à recouvrir la vue et l'ouïe. L'écrivain dit écrire pour les malentendants, car elle souhaite faire entendre une voix inspirée, la voix rédemptrice du Christ ou plus généralement le mystère inhérent à la nature auquel la nature humaine est imperméable. La poétique criarde d'o'Connor apparaît sous de multiples facettes, notamment celle de figures volontairement grossières comme la jambe d'Hulga qui n'est que la métaphore de son âme, aussi cruciale pour elle que la queue pour le paon. C'est dans ce cas au niveau symbolique et visuel que la stratégie esthétique opère, tel « un gros symbole qui vous frappe en pleine face " (Magee, 1987, 59). Mais le diamant de son écriture renvoie également tout un univers sonore qui choque l'oreille.

2 Pour comprendre et entendre les voix de sa prose, il faut, comme le souligne O'Connor à son propre sujet dans un article intitulé «le Roi des oiseaux » $(2009,815)$, « être une domestique tenue d'obéir au cri et à l'œil du moindre de ces princes emplumés ». Comme l'ouïe fine du paon sur le qui-vive (elle en élevait une quarantaine dans sa ferme de Géorgie), l'oreille de l'écrivain du Sud est particulièrement aiguisée par sa cohabitation avec toutes sortes de gallinacés, bizarreries pour la vue et pour l'oreille. C'est tout un monde de bruits qui transparaît en contrepartie d'un portrait à la Jérôme Bosch d'une série de personnages typiques du Sud aux capacités visuelles et auditives défaillantes (Rayben est sourd, Hulga parle peu, la voix de Mrs McIntyre s'affaiblit...). Que dire aussi de ses lecteurs qui potentiellement restent sourds à son 
message et exigent d'elle une voix tonitruante, une voix efficace qui s'exprime sur la page et s'imprime dans les esprits.

3 Cette double technique visuelle et auditive n'est pas sans rappeler les criaillements grotesques du paon qui dévoile toute la beauté de l'univers en faisant bruyamment la roue, suscitant le mutisme de l'observateur. Tel le visiteur en fourgonnette qui, arrivé chez Flannery, voit le paon faire la roue et reste concentré sur «le roi des oiseaux » $(2009,815)$ « comme s'il cherchait à déchiffrer à distance des caractères d'imprimerie très fins ", le lecteur est invité à décrypter les voix de ce petit monde. On est frappé par l'étonnante récurrence de lettres majuscules qui (comme dans les bandes dessinées) représentent les cris des personnages ou des voix animales que le texte laisse échapper à dessein. Cette stratégie de monstration anime un bestiaire sonore, un univers grouillant de vies et de cris, une véritable basse-cour à haute voix.

\section{La voix, véhicule de la révélation}

4 L'essence du mystère de la foi s'incarne dans les voix, véritables véhicules de la révélation. Cris et bruits attirent l'oreille et l'œil vers ce qui se tient près de nous, mais que nous refusons d'entendre. Pour le lecteur réfractaire, la lecture équivaut ainsi à une quête de voix inhabituelles, comme pour rétablir l'unité mythique perdue entre le mot et la chose ou « croire en une reconquête romantique et mystique d'une voix qui émet un souffle de spontanéité, d'émotions et d'immédiateté dans le mot écrit ». Cette explication fournie par Jean-Pierre Martin dans la Bande sonore $(1998,125)$ laisse entrevoir la page écrite par O'Connor comme une tentative de restauration de ces voix. Les nouvelles sont en effet l'occasion de recueillir quelques mimologismes relatifs aux oiseaux notamment, des onomatopées récoltées par l'imagination qui traduiraient un langage divin.

5 L'œuvre laisse apparaître une quête du divin qui nous surpasse, la présence qu'elle convoque est celle du mystère de/dans la voix, souvent présenté de manière symbolique (sous la forme d'un taureau, d'une lune, d'un paon...). Ainsi, celui qui écoute, est-il plus proche de Dieu : «The bull, silvered in the moonlight, stood under it, his head raised, as if he listened- like some patient god come down to woo her-for a stir inside the room » («Greenleaf», 1971, 311)1.

6 Alors que dans "Greenleaf ", Mrs May refuse de prendre en compte cet agent divin, d'autres personnages espèrent encore retrouver cette oralité mythique telle que le révèle Hulga dans ses dialogues imaginaires. Dans "Braves gens de la campagne" $(2009,318)$, elle rêve d'une conversation idyllique et profonde avec le vendeur de bibles. De même, l'oralité revêt paradoxalement un caractère sacré pour le désaxé nihiliste dans la nouvelle intitulée "les Braves gens ne courent pas les rues». Petit garçon respectable, il était chanteur de gospel «à la voix rêveuse». Mais lorsqu'adulte, la communication est rompue avec son interlocuteur, il a recours au meurtre. Le péché le plus lourd serait donc, si l'on en croit ce personnage ambigu, de rester sourd à la voix de l'Autre. C'est ce que Pointer, autre diable "au service" de la révélation semble reprocher à Mrs Hopewell au seuil de sa porte : «Lady, he said, the word of God ought to be in the parlor» $(1971,278)^{2}$. Parallèlement, l'enfant dans « les Temples du SaintEsprit » se sent coupable d'un manque de pratique de la foi lorsqu'elle entend la voix noble du calliope («belle voix » en grec ancien). 
7 Dans les nouvelles d'o'Connor, la voix de Dieu est peu écoutée, vécue comme une habitude contraignante. Les personnages défaillants ou disciples endormis passent par la violence des bruits : corps et esprits sont mis à mal par les voix. Leur surdité réclame un séjour dans la gueule du dragon pour favoriser l'éveil. Ainsi, les histoires invitent à écouter cette voix perdue, pour nous remémorer la voix mythique.

Des voix imparfaites et déformées comme sorties de la gueule d'un dragon en colère sont ainsi le premier stade vers le recouvrement de cette voix mythique. Comme le souligne Steven Connor $(1998,215)$, les voix revêtent quelque chose d'énigmatique, « un conflit entre l'incarnation et le sens », « des moments de rupture dans l'exercice de la voix", "une attention particulière portée à une diction imparfaite ou déformée $»^{3}$. Les personnages à la voix déformée sont comme le paon mâle qui expulse des profondeurs de sa gorge d'ignobles sons en déployant sa queue, "à croire qu'il a reçu par les pattes un choc venu du centre de la Terre, qui lui monte au gosier et qu'il laisse échapper " («le Roi des oiseaux », 2009, 817). À leur tour, les personnages à la voix défaillante, incapables de communiquer subissent des chocs auditifs et corporels pour vaincre leur surdité et leur mutisme.

\section{Faire entendre la voix rédemptrice du Christ}

9 Ainsi, le prêtre catholique dans « la Personne déplacée » s'émerveille-t-il devant la roue du paon qui se déploie simultanément en secouant ses plumes irisées dans de bruyants frissons. Comme Ted Hughes qui pense que les oiseaux possèdent un langage voyant, une voix qui transperce le réel, l'homme en extase ne voit-il pas dans l'oiseau plus qu'un oiseau, le symbole conventionnel du Christ associé à la sainte communion : «le Christ reviendra ainsi », déclara-t-il d'une voix forte et joyeuse (...).» $(2009,357)$ ? A contrario, Mrs McIntyre ne voit en ce volatile qu'une bouche de plus à nourrir préservée par respect pour son mari défunt. D'autres spectateurs ou lecteurs ne retiennent que son infirmité vocale qui fait rire, sa clameur sonore ou d'affreux cris aigus qui font fuir. C'est par la voix du prêtre que Flannery O'Connor a choisi de porter le message (divin) de l'oiseau, car pour lui aussi, l'oiseau est symbolique : "'What a beauti-ful birdrrrd!', the priest murmured» $(1971,225)$. Le roulement ou prolongement exagéré du $\mathrm{r}$ dans «bird " qu'il donne à entendre lors d'exclamations récurrentes, exprime le regret de voir Mrs McIntyre déplorer la présence du charpentier polonais (« the porrrrr man »), de l'oiseau ou de M. Shortley (« arrrrrrr! »). Cette voix rauque qui en quelque sorte rend hommage aux vociférations du paon, appelle par la même occasion la propriétaire à réagir et agir pour le bien. Le détachement des syllabes dans la voix admirative du prêtre n'est que la musique de son âme qui sort de sa gorge pour porter à cette femme un message de rédemption. Elle devrait, comme beaucoup d'autres personnages défaillants, voir la réalité autrement, voir la beauté suffisante du paon, voir l'utilité du travailleur polonais (Mr Guizac) et de Mr Shortley. Dans cette nouvelle, le paon, tout comme ce charpentier réfugié et catholique (figure christique) est rejeté, marginalisé, prétexte à incarner l'irruption soudaine du divin pour racheter l'âme de Mrs McIntyre. Le prêtre évoque un moment de «Transfiguration » que le paon susciterait. Parallèlement, c'est l'écoute ou la lecture du mot « birdrrrd » qui évoque la résurrection de la parole de Dieu aux yeux du prêtre, la transfiguration du mot et de l'oiseau (messager officieux) s'opérant en même temps dans la voix du prêtre (messager officiel). 
D'autres écrits d'O'Connor poussent le vice jusqu'à faire entendre la voix manipulatrice du diable pour tester la capacité de réaction des personnages à aller vers le bien. Dans "les Boiteux entreront les premiers", Sheppard, père de Nortorn qui vient de perdre sa mère, se préoccupe davantage d'un rebelle réfractaire avec lequel il ne parvient pas à communiquer. Rufus Johnson incarne la voix du diable qui incitera l'enfant à se suicider pour rejoindre sa mère et les étoiles vues à travers un télescope. Charité bien ordonnée commence par soi-même: Sheppard apprendra la leçon aux dépens de son fils. L'adolescent se dit possédé par Satan et lorsque Sheppard tente de le raisonner, il ne réalise pas que Rufus incarne lui-même le diable dont il tente de le protéger. La larve $\mathrm{du}$ mal finit par éclore au sein du cocon familial, car le père reste sourd à la tristesse de son propre fils.

11 Dans le roman «Et ce sont les violents qui l'emportent », la voix du diable, « une voix forte, étrange et déplaisante » $(2009,376)$ tente de décourager la croyance de Tarwater en la rédemption. Ainsi, Tarwater noie-t-il un enfant au lieu de le baptiser : « Be a man. It's only one dimwit you have to drown» $(1971,215)^{4}$. La voix du diable est une des «formes que revêt le dragon, ce sont les péripéties de ce mystérieux passage sous son œil, ou dans sa gueule, que se donne pour objet de raconter tout récit un peu profond » $(2009,820)$.

\section{En passant par la gueule du dragon}

Cette citation tirée de l'essai «le Romancier et son Pays» $(2009,820)$ éclaire sur la pertinence de l'usage du terme. En effet, l'étymologie du mot « dragon » est dérivée de derkomai (voir clair en grec). Lire, ce serait donc y voir plus clair en découvrant la Géorgie profonde ou «la gueule du dragon » comme l'a surnommée O'Connor dans « Loin du Paradis ».

13 Passer par la gueule du dragon, c'est pour beaucoup de personnages subir les voix et les actions d'animaux fabuleux et terrifiants. Souvenons-nous du bruit régulier des mâchoires du dragon-taureau qui finit par encorner Mrs May. Passer dans la gueule du dragon, c'est encore se soumettre au comportement déviant de drôles de gargouilles bruyantes ou subir les foudres des furies (les paroles attendrissantes de la grand-mère ne peuvent enrayer la folie du désaxé dans «Les braves gens ne courent pas les rues »). Comme les petits paons nés dans la ferme de Flannery O'Connor qui, d'après celle-ci, parviennent à survivre et ne semblent mourir que de mort violente, les personnages qui survivent sous la plume de l'écrivain peuvent parfois être assassinés ou trompés d'un ultime trait. C'est le cas par exemple de la grand-mère qui constate que "Les braves gens ne courent pas les rues». Agacé par les palabres de celle qui dit le reconnaître comme son enfant (moment hiérophanique pour la grand-mère), le désaxé finit par la tuer de trois coups de revolver dans la poitrine. Le criminel fictif n'est pas sans rappeler le réel vendeur de pieux de clôture, ancien propriétaire de paons qui raconte à F. O'Connor dans «le Roi des oiseaux » $(2009,820)$ comment il a fini par les manger. En effet, les volatiles l'empêchaient de penser tellement leur voix stridente était insupportable : «au printemps, on ne s'entendait plus penser. Dès qu'on haussait la voix, ou même avant, ils ouvraient le gosier ». O'Connor, elle, laisse la vie sauve à ses paons. Si le paon crie à l'aide, c'est qu'il est en proie à de violents rêves : « souvent, ils se réveillent pour crier : 'au secours! au secours!' alors de l'étang, de la grange, des arbres autour de la maison s'élève un chœur de supplications : 'Lii-on-lii-on !/Mii-on- 
mii-on !/Eii-i-oi-i-i-oi !/Eii-I-oi-I-I-oi !' (« le Roi des oiseaux », 818). L'auteur laisse donc le dernier mot à ses paons, car ils incarnent la voix divine, mais elle met à mal les personnages qui refusent d'écouter.

Passer par la gueule du dragon, c'est aussi cohabiter avec de drôles d'oiseaux, femmes acariâtres (Mrs May ou Ms Cope) ou enfants mutins (Bailey, Rufus Johnson, Temple One et Temple Two, Julian). Les péripéties du récit, ce sont aussi ces moments où personnages et lecteur sont confrontés à toutes sortes de voix de volatiles ou de noms d'oiseaux. Dans le monde animal répertorié, le dragon désigne un pigeon fantaisie au bec fort venant d'Angleterre. Dans sa fiction, point de pigeon (au sens propre tout du moins), mais beaucoup d'autres volatiles qui peuvent également se parer des plumes du dragon, car l'auteur collectionne les monstres à plumes : paons estropiés, cygnes à œil unique, poules qui marchent à reculons, canards et dindons aussi laids que bruyants. Les bruits produits par tous ces drôles d'oiseaux, oiseaux rares ou oiseaux de malheur, vrais ou faux paons, poules caquetantes ou prodiges, marginaux, désaxés, handicapés, pauvres, noirs, pauvres diables, prophètes blancs fous et illettrés, commères, enfants pervers, hystériques ou victimes, sourds au propre comme au figuré, sont un passage obligé de la lecture.

La voix du mystère s'incarne dans cette sonate de voix contradictoires. Toute sa vie durant, O'Connor fut à la recherche de paons à trois jambes ou trois ailes (« le Roi des oiseaux ", 2009, 811). Comme ses personnages insolites, ces bêtes curieuses qui attirent l'attention poussent ceux qui les rencontrent à entrer dans la gueule du dragon pour comprendre le sens de la vie. Le dragon guide les êtres humains en perdition vers une voix qui les invite à corriger leurs erreurs. Dans «C'est peut-être votre vie que vous sauvez », Lucynell Crater est sourde et muette, mais la couleur de ses cheveux et des ses yeux comparée au bleuté du cou du paon fait d'elle une créature inspirée de Dieu. June Star et John Wesley dans « les Braves gens ne courent pas les rues » sont comparés à de bruyants macaques, leurs paroles incessantes trouvent leur pendant dans le rire démoniaque du bandit de grand chemin, le dragon auquel ils sont confrontés sur leur route. De même, Mr Head le grand-père de Nelson dans "le Nègre factice", est confronté à ses propres préjugés racistes lorsque son fils s'attarde auprès d'une femme noire. Son attendrissement qui est prélude à une révélation est écourté par les propos insultants de Mr Head que le texte rend effectivement audibles: "And standing there grinning like a chim-pan-zee while a nigger woman gives you direction. Great Gawd! » ("The Artificial Nigger », 1971, 263) .

Puis c'est le grand-père qui se retrouve à faire le singe : « Mr Head, hunched like an old monkey on the garbage can lid, decided if Nelson didn't wake up soon, he would make a loud noise by bamming his foot against the can » $(1971,264)^{6}$. Mr Head devient étranger à son petit-fils, passant d'un comportement singé bruyant et raciste au mutisme de la tortue. Lorsqu'il sort de sa cachette avec lenteur, honteux, il constate sans intervenir que Nelson est en conflit avec une femme noire dont il a malencontreusement cassé la cheville.

17 Dans « les Boiteux entreront les premiers », Sheppard essaie vainement de comprendre la part obscure de Rufus, adolescent boiteux en maison de redressement. Conseiller bénévole, il espère entrer dans la tête de Rufus pour le changer comme s'il voulait volontairement faire un détour par la caverne du dragon au lieu de s'occuper en priorité de son propre fils, orphelin de mère: «He imagined his (hypnotic) voice penetrating to the boy in the black caverns of his psyche» $(1971,472)^{7}$. Son excès 
d'altruisme n'empêchera pas Rufus d'agir pour le mal. Le père est resté sourd aux avertissements (sonores) de son fils : la nature de la voix de l'enfant annonce pourtant son suicide. Lorsque Sheppard lui demande s'il serait plus heureux chez les Johnson, son fils lui rétorque: "I don't know', the child said lamely" $(1971,447)^{8}$. Sa voix gauche et bancale annonce discrètement que les derniers seront les premiers («The Lame Shall Enter First »). La manière de la voix dévoile l'intrigue : Norton est l'un des handicapés (il a perdu sa béquille maternelle) à entrer le premier au paradis, en se pendant dans le grenier.

Passer par la gueule du dragon, c'est accepter d'entendre, de voir la révélation si douloureuse soit-elle. Le dragon ne désigne-t-il pas aussi la huitième plus grande constellation qui symbolise la vigilance renforcée par une vue perçante et une ouïe fine. Dans le ciel étoilé, la constellation du dragon est visible toute l'année, elle ne se couche jamais. Parallèlement, dans chaque histoire, on peut dire aussi que les formes vocales que revêt le dragon sont inextinguibles, car elles ne se taisent jamais. Les personnages sont sans cesse confrontés à la souffrance et à la vérité.

Le dragon, c'est aussi le gardien du mystère des voix du sud, rite de passage d'où les personnages reviennent transfigurés. On offre par exemple, à Mrs May dans sa ferme en Géorgie profonde (comme au Jason mythologique en Géorgie caucasienne) l'occasion (refusée) d'apprivoiser un taureau merveilleux. L'animal divin fait aussi écho à l'ânesse de Balaam du livre des Nombres (22) qui reconnaît mieux que son maître l'action de Dieu.

\section{Les voix animales, l'exemple des galliformes (paons, poules et dindes)}

Taureau, paon, dragon, dindon, puma, singe, tortue, poule, cygne : voici une liste non exhaustive de tous les animaux qui ont une place de choix dans le bestiaire de F. O'Connor, dans ses écrits théoriques et fictifs, mais aussi pour certains, dans sa vie. On remarquera au passage que la plupart font partie des quatre-vingt-huit constellations célestes (le cygne est la croix du nord, l'étoile du paon est la constellation de l'hémisphère sud...). Voix et visions vont de pair dans son écriture. Lorsqu'on la questionne sur son style, elle répond en utilisant les métaphores animales du babouin et de la girafe pour désigner les voix des écrivains contradictoires : "J'imagine que ce que vous déclare une semaine la girafe est contredit la semaine suivante par le babouin " ("Mystères et manières ", 2009, 842). Lorsque Margaret Turner lui rend visite dans sa ferme en mai 1960, elle remarque le vacarme chaleureux des paons ou le klaxon tapageur des oies (Magee, 1952, 41-46).

On se souvient en priorité de ses personnages grotesques comme les acteurs principaux de ses nouvelles, mais O'Connor souligne la primauté accordée aux animaux et à leurs voix. Je sélectionnerai ici le cas du paon et de la poule qui se croisent de manière évidente dans ses nouvelles comme dans sa biographie. Ne s'est-elle pas amusée à coudre des vêtements pour volailles: « un bantam gris, baptisé colonel Essbert, a eu ainsi droit à une tunique en piqué blanc, avec un col de dentelle, fermée dans le dos par deux boutons» («le Roi des oiseaux», 2009, 812). Lorsqu'elle décrit le paonneau, il apparait encore plus grotesque que ses personnages : « cette aigrette ressemble d'abord à une antenne d'insecte, puis à la couronne de plumes d'un Peau-Rouge » (« le Roi des 
oiseaux ", 2009, 815). Pour sa part, le cri du paon adulte est strident et répétitif, mais lorsqu'il fait la roue, c'est un silence gracieux qui fait irruption dans son monde :

La nuit, ils criaient comme un chœur grec qu'on allait égorger. Le jour, ils dévoraient les fleurs de sa mère; et pendant des heures, l'après-midi, elle restait assise sur l'escalier de la cour, à étudier les lois de ce monde si éloigné du nôtre, qui peut-être lui révélait quelque chose du nôtre. (Citati, 1992, 238).

Les voix des animaux, qu'ils soient cochons, dindons, ou paons véhiculent le sens du mystère. C'est de la matière et de la manière des voix que la fiction d'o'Connor est faite, elle opère à travers les sens, la vue, et l'ouïe souvent trop négligée par les critiques. Les voix grotesques sont déformées pour être mieux entendues. O'Connor utilise des voix animales excessives et violentes pour aider les personnages à revenir au réel. Les voix naturellement grossières ou caricaturales (celle du paon femelle par exemple) s'intègrent facilement à ce monde sonore décrit longuement par Flannery O'Connor dans « le Roi des oiseaux» $(2009,817-818)$ :

Li-ooo-ii ! Cri de mélancolie pour les mélancoliques, d'hystérie pour les hystériques. À mes oreilles, il a toujours retenti comme un hourra qui saluerait une parade invisible. La femelle se livre rarement à de pareils éclats. Le cri qu'elle pousse ressemble au braiment de la mule - hihan hihan, i-aan. (...) Au printemps et en été, (...) le mâle (...) penche la tête en arrière pour lancer une série de sept ou huit cris successifs comme s'il n'était pas sur la terre de message plus urgent à capter que le sien. Le soir, ces appels prennent un ton mineur, et l'air en vibre à une lieue à la ronde... (...) De tous ces perchoirs partent des appels et des réponses dont retentit la nuit.

O'Connor affectionne les poules rares et les paons tout comme des voix marginales dérangeantes. Par exemple, le criminel dans «Les Braves gens ne courent pas les rues » est un drôle d'oiseau antipathique. Tout comme le paon, il choisit un endroit ou des mots qui dérangent. Son refus de compassion qui transparaît dans ses paroles est semblable au cri strident du paon qui manifeste sa faim ou tue pour ne pas être tué. Ses paroles finales après son crime résonnent aussi comme un "Léon Léon » absurde et victorieux : "La ferme, Bobby Lee! dit le désaxé. Y a pas de vrai plaisir dans la vie.» $(2009,206)$. Cette voix marginale et dévastatrice semblable au corbeau carnivore qui crie lors d'un décès en Catalogne ou en Auvergne ${ }^{9}$, semble éteindre à jamais les cris hystériques des enfants lors de l'accident de voiture préalable à la rencontre mortelle ou les fous rires et gloussements des petites filles dans « les Temples du Saint-Esprit ». Le "Léon Léon » victorieux de l'assassin (paon mâle) répond dans un écho inversé au « Hihan Hihan » ou aux appels au secours de la grand-mère (paon femelle ou dinde) qui caresse encore l'espoir vain de ne pas laisser trop de plumes dans cette mésaventure.

Autre oiseau qui faisait partie intégrante de ce monde bruyant : la poule qui caquette et ne vole pas. Le poulailler est pour O'Connor, un refuge contre les mondanités. Les bruits, sons et voix, grains des gosiers sont pour elle la preuve de la vitalité du monde. Dans «Et ce sont les violents qui l'emportent », Francis Mario Tarwater en fait les frais lorsqu'il se demande comment gérer la propriété qu'il croit désormais sienne quelques minutes après la mort de son grand-oncle. Seul le maître d'école peut venir réclamer la terre et il pense alors que " le seigneur l'enverra peut-être ailleurs ». S'attendant à une confirmation venant d'en haut, il est surpris par le bruit d'une simple poule : « he held his breath as if he were about to hear a voice from on high. After a few moments, he heard a hen scratching between him under a porch » $(1971,12-13)^{10}$. C'est un signe d'en bas, de la basse-cour qui vient à lui, comme pour lui dire de redescendre sur terre. Le signe de Dieu qu'il attend n'est que le grattouillis d'une poule qui l'invite à se 
préoccuper en premier lieu de l'enterrement de son grand-oncle dont le corps attend sagement sur la chaise de cuisine. La poule néanmoins lui montre la voix/voie...

Dans « les Braves gens ne courent pas les rues ", ce sont les cris de la grand-mère avant sa mort et ceux de Bailey qui rappellent les cris «d'une vieille dinde desséchée qui piaille pour avoir de l'eau : “Bailey, mon fils, Bailey, mon petit !" cria-t-elle » (2009, 205). Poule et poussin se répondent ${ }^{11}$. La grand-mère auparavant vaniteuse, tenant à son chapeau comme un paon à ses barbes, se comporte comme une mère poule/paonne qui tente de rassembler ses petits à l'approche du danger. L'ultime petit qu'elle tente ensuite de sauver, c'est le désaxé qu'elle dit reconnaître comme l'un des siens. La valeur de cette supplication affective ou puissant coup de trompe n'est pas reconnue comme telle par le bandit. La dinde n'est qu'une version déclassée du paon et les autres dindons de la farce ou faux paons du clan familial ont déjà tous péri, son signal d'alarme ne peut être entendu. Comme la voix du paon qui excite le rire (par sa ressemblance avec les hennissements d'une mule), la voix de la grand-mère suscite chez le désaxé un élan d'humour noir. Après l'avoir assassinée, il déclare : « ç'aurait pas été une mauvaise femme, dit le désaxé, si y avait eu quelqu'un pour la suriner à chaque minute de son existence. Marrant! dit Bobby Lee » $(2009,206)$. La voix de la grand-mère ne lui est d'aucune aide pour faire comprendre son regain de compassion : " mais vous êtes un de mes petits! Vous êtes un de mes enfants à moi!» $(2009,205)$. Ses paroles restent incomprises comme les vociférations du paon qui le trahissent toujours sans jamais lui donner la possibilité d'exprimer vérités et nuances par le chant. Le désaxé la réduit à la compassion inutile et au silence éternel comme un paon résigné qui aurait compris que sa beauté ne réside ni dans son apparence extérieure ni dans des paroles éraillées, mais dans le silence.

\section{Les personnages : des voix marginales et vicinales}

Mais «il y a les voix, quantité de voix. Flannery O'Connor n'est jamais rassasiée d'écouter les paroles de ses personnages : (...) elle sait que le récit est, en premier lieu, une sonate de voix qui interrogent et répondent». Face à ces propos de Citati (1992, 247), on comprend mieux comment O'Connor invite le lecteur à écouter ses histoires pour y entendre le divin perdu ou caché dans le quotidien. Faire marcher les poules caquetantes à reculons, se pâmer devant la roue du paon qui fait frémir ses plumes, montrer l'extraordinaire dans l'ordinaire, déceler le mystère de la présence divine dans un défaut de prononciation : ses nouvelles se tournent vers l'oralité, non pas comme un espace restreint, mythique et sécurisant, mais pour réconcilier les hommes avec le sens de l'existence. L'oralité produite par les personnages (bruits, défauts d'élocution...) est symptomatique de sa volonté de donner voix aux marginaux illettrés, une tentative, selon Geneviève Brisac $(2002,41)$ « d'éterniser la langue des prophètes fous et des pauvres diables blancs du sud ». Paradoxalement, son écriture transporte leurs voix sous une forme dégradée, montrant la nécessité d'une parole éclairante :

L'écrivain sudiste, sorte de quasimodo des champs de maïs, la bouche pleine de syllabes à moitié avalées (...) écoute l'étrange langage qui jaillit de cet endroit bizarre qui est chez elle, (...) passe par le langage des désaxés pour comprendre son mystère. (Brisac, 2002, 42-47). 


\section{Transcender les voix du sud (accents et idiomes régionaux)}

Dans deux entretiens donnés à Harvey Breit (1955, dans Magee, 1987, 9) et Granville Hicks (1962, dans Magee, 1987, 82), Flannery O'Connor concède l'importance incontournable de l'accent du sud, d'une oreille accoutumée au parler géorgien. Mais cet idiome régional ou ces voix vicinales sont utilisées à bon escient pour suggérer ce qui les transcende. "Quand un personnage du sud parle sans se soucier de son rang, c'est toute la vie du sud qui s'éveille aux accents de sa voix » (" Mystères et manières », $2009,915)$ et pour « révéler le mystère d'un être », il est habile de « lui faire parler une langue qui est la sienne» («Mystères et manières», 2009, 866). Au milieu des caquètements de poules et des cris animaliers, son univers imaginaire laisse donc entendre les voix de son terroir : les commérages, les cris de désespoir, la voix rauque d'un assassin, des expressions vernaculaires, des voix qui ne savent pas s'arrêter, comme si la bêtise humaine apparaissait de manière audible/auditive. La qualité vernaculaire ou vicinale des paroles n'est pas liée au rang social ou à la couleur de peau, mais combinée aux défauts de prononciation et aux fautes de grammaire, elle révèle davantage la malveillance, les intentions erronées ou un manque d'âme qui revêtent une dimension universelle. Ainsi les formes que prennent les élocutions de Mr Head et de son petit-fils ressemblent à du créole ou "pidgin English »: "We eaten before we left! » (Mr Head, 1971, 256), « 'you were the one holding the sack' Nelson said 'I would have kepaholt of it' » $(1971,261)^{12}$.. Mais les paroles de Mr Head trahissent avant tout le racisme qu'il donne en modèle à son petit-fils. Les péripéties de la nouvelle le mettent face à ses défauts comme la petite fille dans « les Temples du Saint-Esprit », qui, tel un paon qui envie le chant harmonieux du rossignol hors de sa portée vocale, reconnaît sa méchanceté et demande l'aide de Dieu: " hep me not to be so mean, she began mechanically. Hep me not to give so much ass. Hep me not to talk like I do » (1971, $247)^{13}$.

\section{Le talon d'Achille vocal}

La plupart des personnages semblent avoir un talon d'Achille vocal, les voix sont déformées et défaillantes. Beaucoup de personnages demandent aux autres de se taire, d'où les bouches qui sont constamment fermées, tordues ou distendues comme des rictus figurant un manque de communication, ou des difficultés d'élocution. Robert Jackson $(2005,87)$ voit un travail de rédemption derrière toutes ces voix imparfaites. O'Connor utiliserait donc toute une panoplie de voix faussées pour faire entendre le mystère. En effet, comme Ruller dans « le Dindon », les enfants mesquins de la plupart de ses nouvelles sont amenés à écouter la voix puissante de Dieu. Leurs mots d'esprit apparaissent, ainsi que leurs invectives, ou leurs paroles grossières mais la langue se retourne toujours contre le personnage ainsi pris en défaut.

Dans « la Personne déplacée », la voix aiguë enthousiaste et décidée de Sulk (jeune Noir qui travaille pour Mrs McIntyre) annonce à la propriétaire de la ferme son intention de se marier avec une réfugiée blanche polonaise de seize ans. Mais la tonalité de la voix baisse lorsqu'il comprend à quel point Mrs McIntyre est réfractaire à son projet : «la voix aiguë sembla fuser comme un jet sonore puis retomba quand il vit le visage de Mrs McIntyre " (2009, 351). Le manque de compréhension et de compassion de la fermière qui traite de «monstre » monsieur Guizac, l'oncle de la réfugiée polonaise, est confronté à sa propre monstruosité lorsque le Polonais «venu pour (les) racheter» 
$(2009,357)$ meurt quelque temps plus tard sous son tracteur: " elle avait entendu le petit bruit de la colonne vertébrale du Polonais quand la roue du tracteur avait passé dessus» (364). Refusant d'écouter les voix suppliantes du jeune noir et de celle de Mr Guizac, bruits et silence de mort s'offrent en dernier recours à la fermière : après une dépression nerveuse, la fermière perd la vue et la parole.

Dans « le Nègre factice », l'expression utilisée par la femme noire bousculée par Nelson, petit-fils de Mr Head annonce la déliquescence du grand-père. En effet, la faute de prononciation dans « He's a juve-nile deliquent! » $(1971,265)$ «C'est un déli (n) quant juvénile!» $(2009,273)$ ou l'omission du «n' dans «deliquent » reflète davantage la déliquescence du grand-père que la véritable « délinquance » du petit-fils. Ici, l'énigme que cette erreur de prononciation laisse entendre est résolue lorsque Mr Head se liquéfie de peur. Il s'imagine transformé en une substance liquide alors qu'il a perdu la considération de Nelson : "The old man felt that if he saw a sewer entrance he would drop down into it and let himself be carried away $(1971,267)^{14}$. Les consonnes liquides


l'impression de liquéfaction ressentie par Mr Head. L'omission de la lettre dans «deliquent» fonctionne comme la faute d'orthographe dans «Chrustian» («Good country people»). Dans «I know you believe in Chrustian service » ${ }^{15}$, phrase de Manley Pointer, faux vendeur de bibles, adressée à la mère d'Hulga, ce sont les intentions maléfiques du fétichiste athée (un vrai chrétien saurait prononcer le mot) qui sont trahies par sa voix. C'est aussi le manque de foi de la famille Hopewell qui la conduira à sa perte (la perte d'une jambe à cause de leur foi « rouillée ", rusted).

31 Dans tous ces défauts de prononciation résonne une voix enkystée adressée à un lecteur qui n'est pas toujours certain de bien entendre. C'est ce que Jean-Pierre Martin (1998, 112-113) souligne :

L'oreille ne s'autorise d'aucune parole (...). Elle doute. Elle n'est jamais sûre de bien entendre. Elle est surtout sensible aux ratages, au manque d'assise des paroles, à l'incertitude du langage, à la glu des mots.

C'est ce manque d'assise des paroles qui travaille la nouvelle intitulée « Les braves gens ne courent pas les rues » dans laquelle le désaxé ébranle la langue et la syntaxe. À titre d'exemple, après l'accident de voiture, il déclare "I see you all had you a little spill » $(1971,126){ }^{16}$. La polysémie du verbe "spill» en anglais qui signifie "chuter», " renverser ", mais aussi « dévoiler » ou " déballer » trahit dans la voix du criminel en cavale ses intentions : il tuera les accidentés, de peur que ces derniers aident la police à les localiser. Autre mot-dragon à plusieurs têtes, le mot plurivoque "reckernized" prononcé par le même personnage dévastateur dans «It would have been better for you, lady, if you hadn't reckernized me » $(1971,127)^{17}$. Dans « reckernized » au lieu de "recognized" (reconnaître), on entend la vraie nature du désaxé («wrecker», destructeur) et le massacre des innocents extirpés de l'épave de la voiture (" wreckage »). Cette fausse note dans la voix pervertit les codes de la langue, mais dit plus que le mot originel. La déformation phonétique peut passer pour un accent du sud, mais transcende cette simple explication régionale en fonctionnant comme un stigmate.

Les néologismes dans la voix montrent moins la richesse de la langue que tous les points morts auxquels se heurtent les personnages. Les paroles servent moins à communiquer et résoudre qu'à sceller un destin funeste. Par exemple, dans l'expression utilisée par le désaxé, «I pre-chate that, lady » $(1971,128)^{18}$ qui est censée remercier la 
grand-mère du compliment qu'elle vient de lui faire sur sa bonté, on peut entendre un idiome local «I appreciate that », mais aussi la violence qui a la primauté puisqu'en proférant ces paroles (pre-chate se rapproche de pre-chat, discussion préalable), le désaxé dessine un cercle avec l'extrémité de son revolver. Inutile de parlementer, ou de prêcher la bonne parole (pre-chate propose un écho visuel et phonétique à preach), le désaxé n'est là que pour régler des comptes. De même, lorsque la grand-mère propose au désaxé d'aller voir dans la valise de Bailey s'il peut lui prêter un tee-shirt, il répond : " I'll look and see terrectly, » $(1971,129)^{19}$. Le mot terrectly en plus de son sens dialectal (soon) représente un mariage phonétique arrangé entre directly et terribly: en effet, le criminel se servira lui-même en dépouillant Bailey de son tee-shirt après l'avoir tué. C'est Bobby Lee, l'associé qui reviendra quelque temps plus tard arborant le tee-shirt aux perroquets bleus.

En plus des phonogrammes qui dévoilent la nature et les intentions dans la voix des personnages, on peut entendre des clichés dans les discours des femmes qui expriment à mauvais escient leurs avis sur tout. Décidément chez O'Connor, soit on parle trop ou trop fort, soit on en dit peu ou l'on se mure dans le silence. Les exemples de voix défaillantes sont légion. Les voix se fêlent (Johnson, Bailey, le vieux dans «le Géranium »), hurlent, jubilent (Johnson, les trois sauvageons), insultent, la communication se bloque, les mauvaises langues fourchent ou mentent, caquètent (" yaketty yaketty yak» dit Rufus Johnson), les personnages parlent pour ne rien dire ou chantent à tue-tête.

\section{Chansons, blasphèmes et onomatopées}

Rufus Johnson dans "Les boiteux entreront les premiers " pousse la chansonnette en battant la mesure. Le rythme irrégulier de ses pas donne voix à son handicap physique et à ses déséquilibres psychologiques: "' Gonter rock, rattle and roll' he sang 'Gonter rock, rattle and roll'. Can't please that woman, to save my doggone soul ». He began to move around, stamping the good foot down.» $(1971,456)^{20}$. Les paroles de Rufus reprennent celles de la chanson de Jesse Stone «Shake Rattle And Roll» (1954), ce qui atteste de leur qualité dramatique. Le parallélisme entre les deux chansons dévoile les futures intentions maléfiques de Rufus. Le rythme jazzy et endiablé des paroles de l'adolescent dévoile son âme perdue («doggone soul»). Plus loin, Rufus s'improvise encore chanteur à la voix aiguë, lorsqu'il déclare que seul le vrai Jésus pourra le sauver : "Jesus'll save me, not that lying stinking atheist, not that... ». " 'The lame'll carry off the prey' he screeched, but his voice was muffled inside the car » $(1971,480)^{21}$. Comme pour les paons dans la ferme d'o'Connor ou comme le désaxé en fuite, c'est un cri (blasphématoire) qui a le dernier mot.

Le drame des personnages peut être chanté et accompagné d'autres voix d'inspiration sacrée ou blasphématoire. Dans " les Temples du Saint-Esprit », Joanne et Susan, deux jeunes filles qui se surnomment "Temple One" et "Temple Two", mais qui ne connaissent pas les Saintes Écritures chantent une chanson à des jumeaux pour se moquer d'eux, "a hillbilly song that sounded half like a love song and half like a hymn » $(1971,240)^{22}$. Cet hymne à la bénédiction est à son tour mal interprété par les deux garçons qui le prennent pour une chanson juive. Cette chanson chrétienne offerte en paratexte exige de ces jeunes gens que "la foi pallie les faillites des sens" («Praestet fides supplemetum, Sensuum defectui», 1971, 241), révélant leur faiblesse 
spirituelle. Blasphème et sacré fonctionnent à l'unisson comme le reconnaît Antonio Di Renzo (1993, 148), car, «dans la cathédrale grotesque des écrits de Flannery O'Connor, le rire rauque des gargouilles ne perturbe pas le ton solennel de l'orgue. L'orgue lui-même résonne et rit $\aleph^{23}$.

Selon la même mécanique musicale, de nombreuses voix parlent en contrepoint des personnages. C'est le cas des mots en italiques qui trahissent les personnages : somebody dans «Greenleaf » montre l'incapacité de Mrs May à écouter la voix de Dieu incarné par le taureau : «It must be somebody's bull » $(1971,314)^{24}$. La fermière se sent assaillie par des bruits de mâchoires, de mastication, jappements (steady chewing, munching, yapping, 1971, 311). Mrs May elle-même produit une sorte de hennissement (" horse wheeze ", 1971, 327).

Les onomatopées jouent le même rôle de contre-voix. Ainsi, le mot «pop » dans « Le Géranium » est-il prononcé par un homme noir qui invite le vieux Dudley à ramasser le pot cassé : « why don't you, pop? » $(1971,14)^{25}$. Encore une fois, il faut sans doute y voir un jeu polysémique dans la voix du voisin. «Pop » signifie à la fois « vieux », mais aussi « mort soudaine ». Ainsi, la phrase frise la conversion grammaticale. On peut s'amuser à interpréter la question de différentes manières, toutes prêtes à sortir de la bouche du personnage anonyme: "et bah vieux, pourquoi tu'l fais pas?», "pourquoi tu n'exploses pas de colère ? » ou « t'as qu'à passer l'arme à gauche » (« why don't you pop off? »). La voix explose : l'onomatopée ou la bulle qui éclate valent mieux que de longs discours. C'est au tour du vieux Dudley raciste et indiscret d'être rejeté, de corriger ses vues et de réparer les pots cassés. Par cette stratégie mimologique généralisée qui (se) traduit par des appels récurrents à la sagesse, « ces petites phrases (...) livrent aussi le contenu du message » divin (Perbosc, 1988, 26).

\section{Un festival sonore : bruits et bulles, cris et phylactères}

L'amateur de nouvelles, dans sa vie comme lors de ses lectures, est sollicité par toutes sortes de bruits. O'Connor voit dans le cri du paon des acclamations suscitées par une parade invisible (« le Roi des oiseaux », 2009, 817) et souligne dans ses écrits théoriques la qualité carnavalesque de la vie. Ce que la plupart des critiques confirment à l'excès, notamment Anthony Di Renzo (1993, 202) qui entend dans l'œuvre d'o'Connor une transcription de palabres venant d'un bus bondé :

Toute grandeur ou clarté disparaît dans une confusion de farces, grommellements, plaisanteries, querelles. C'est comme un marché, un carnaval de bruits. L'étrange multiplicité des voix dans la fiction d'o'Connor explique sa fascinante polyphonie ${ }^{26}$.

Les autres voix dans les nouvelles d'O'Connor sont en effet multiples, ce sont des soupirs à peine audibles, des vieilles dames qui pépient, des bruits de rue, des sons grinçants inintelligibles, des claquements de porte, d'horribles bruits, des gémissements, des propos incantatoires et maléfiques, pour ne citer que quelques bruits glanés à l'écoute de deux nouvelles («le Géranium» et «Braves gens de la campagne »). Ce carnaval sonore qui joue sur une panoplie de sons indéterminés serait le signe d'un monde en manque de sacré.

41 Ou bien doit-on en effet comprendre que Flannery O'Connor passe par les formes séculaires de la voix pour tenter de créer « des interférences en brouillant la qualité graphique du texte pour (...) obliger ses lecteurs à écouter ». Jean-Pierre Martin (1998, 127) guide notre lecture ici vers d'autres voies. À l'instar des mots en italiques qui 
prennent la voix des personnages à témoin, sont en jeu d'autres manifestations graphiques de la langue. Les mots en lettres majuscules reprennent la technique du lettrage si prisée dans les bandes dessinées. La voix se place alors dans des endroits inattendus. Dans "Tout ce qui monte converge", la femme noire vêtue du même chapeau que la mère de Julian sent sa colère monter, elle est ainsi décrite: "the downward tilt of her large lower lip was like a warning sign: DON'T TAMPER WITH ME» $(1971,415)^{27}$. Les cris s'entendent et se voient simultanément comme dans les bulles ou phylactères. Traditionnellement et étymologiquement, les phylactères étaient faits pour contenir le mal, comme si la bulle pouvait retenir la violence des gestes. Et en effet, la voix est refoulée pour être remplacée par un grommellement silencieux (muted growling, 1971, 416) et un poing qui explose au visage de la mère raciste. MariePierre Pasquier $(1988,47)$ explique en ces termes la mécanique de l'écriture d'o'Connor: " on souffle une bulle de savon, on la contemple une seconde dans sa plénitude irisée et la seconde d'après, elle pète ». Autre exemple frappant du principe, l'excitation puis la reconnaissance de la gravité de l'accident de voiture par des jeunes adolescents dans « les Braves gens ne courent pas les rues » rendue à trois reprises par la grosseur des lettres : «We've had an ACCIDENT » $(2009,125-26)$. L'accident mènera à leur assassinat. Dans la Bande sonore, Jean-Pierre Martin $(1998,36)$ explique que l'oralité dans la prose tente de faire revivre la lettre morte en se faisant l'écho des voix et des bruits du monde. Toutes ces voix animales et humaines, ces bruits du monde se font écho dans la syntaxe d'o'Connor telle une composition sonore dans un ensemble hétéroclite, mais parfaitement orchestré.

\section{Une écriture à hautes voix}

42 La nouvelle, forme littéraire transmise oralement à l'origine, ressuscite l'intimité de la voix ou de la Parole divine. D'autres sons émergent : les bruits des animaux dont les cris rappellent la vivacité du monde, les voix des personnages qui trahissent leur aveuglement face au mystère. Même si les personnages ou les lecteurs restent sourds à la Parole divine, l'écriture d'o'Connor réussit à accoutumer l'oreille au mystère de l'existence, suscitant l'écoute de ce que Roland Barthes dans le Plaisir du texte $(1982,104)$ définit comme une "écriture à haute voix ", " un texte où l'on puisse entendre le grain du gosier ».

43 Pour écouter et entendre cette écriture à hautes voix, le lecteur doit passer par le gosier du dragon sous la plume de paon de Flannery O'Connor: sa joie muette, mais vocale trouve un porte-voix dans toutes ces créatures et créations sonores. Comme une reine qui comprend le langage sacré des oiseaux et le vrai langage du monde, tel Salomon, souverain biblique qui converse avec les oiseaux, à la façon de Grégoire le Grand les yeux rivés sur les enluminures qui écoute le Saint-Esprit murmurant à ses oreilles sous la forme d'un oiseau (Perbosc, 1988, 34), F. O'Connor sait écouter le mystère du monde qui s'incarne dans toutes les manières des voix. Par cette conception quasi naturaliste du langage, par le grain rugueux des voix animales, régionales ou marginales, le texte rend sensibles aux mystères du monde. De prime abord, ce n'est pas « une parole claire » que l'auteur réussit à faire émerger même si elle affirme vouloir entendre « des voix, du moins des voix distinctes ». Elle concède ne percevoir que «des murmures confus, grinçants, comme des grondements de chats amoureux » (Brisac, 2002, 109). Ce sont tous les signes d'une présence au monde, signes 
des oiseaux, mi-voix, ratages et allitérations bruyantes que le lecteur est invité à écouter, ravivant dans le temps de son exploration visuelle et auditive ses réflexes d'enfant ou de poète prêt à interpréter les onomatopées des cris des dindons, le souffle divin produit par l'exquis frémissement des plumes de paon sous la plume d'une magicienne.

\section{BIBLIOGRAPHIE}

BARTHES, Roland, 1982, le Plaisir du Texte, Paris, Seuil, coll. « Points Essais ».

BREIT, Harvey, 1955, A Goog Man Is Hard to Find, Transcript of the Galley Proof program filmed by WRCA-TV (NVC), New York, dans Rosemary M. Magee (ed.), Conversations with Flannery O'Connor, coll. "Literary conversation Series", Jackson, University Press of Mississippi, 5-11.

BRISAC, Geneviève, 2002, Loin du Paradis, Paris, Éditions de l'Olivier, coll. « Petite bibliothèque de l'olivier ».

CITATI, Pietro, 1992, Portraits de femmes, Paris, Gallimard.

CONNOR, Steven, 1998, Echo's Bones: Myth, Modernity and the Vocalic Uncanny, in Michael Bell and Peter Poellner (eds), Myth and the Making of Modernity. The Problem of Grounding in Early Twentieth-Century Literature, Amsterdam, Atlanta, Rodopi, pp. 213-237.

DELARUE, Paul et TÉNÈZE, Marie-Louise, 1976, le Conte populaire français. t. III. Contes d'Animaux, Paris, Maisonneuve, p. 17-29.

DI RENZO, Anthony, 1993, American Gargoyles: Flannery O'Connor and the Medieval Grotesque, Carbondale (ILL), Southern Illinois University Press.

JACKSON, Robert, 2005, Seeking the Region in American Literature and Culture, Modernity, dissidence, Innovation, Baton Rouge, Louisiana State University Press.

HICKS, Granville, 1962, A Writer at Home with her Heritage, Saturday Review Magazine, 45, 22-23, dans Rosemary M. Magee (ed.), 1987, Conversations with Flannery O'Connor, University of Mississippi, coll. « Literary conversations series », 81-85.

JAMAIN, Claude, 2002, la Voix sous le texte, Angers, Presses de l'Université d'Angers [Actes du colloque d'Angers, 4-5 mai 2000].

MAGEE, Rosemary M. (ed.), 1987, Conversations with Flannery O'Connor, coll. "Literary conversation Series", Jackson, University Press of Mississippi.

MARTIN, Jean-Pierre, 1998, la Bande sonore, Paris, Éditions José Corti.

o'ConNoR, Flannery, 1971, The Complete Stories, London, Boston, Faber and Faber.

o'CONNOR, Flannery, 2009, Euvres complètes, Paris, Gallimard.

PASQUIER, Marie-Pierre, 1988, Un paon pour ange gardien, les Cahiers du Grif, vol. 39, p. 39-48.

PERBOSC, Antonin, 1988, le Langage des bêtes, mimologismes populaires d'Occitanie et de Catalogne, «Classiques de littérature orale », textes édités par Josiane Bru, Carcassonne, Garae/Hésiode. 


\section{NOTES}

1. «Le taureau, que le clair de lune revêtait d'une robe d'argent, était immobile sous la fenêtre, tête dressée comme s'il attendait - tel un dieu patient descendu sur terre pour courtiser une mortelle - que lui parvînt quelque signe de sa présence " $(2009,533)$. Toutes les citations de l'article en anglais sont des extraits de Flannery O'Connor, 1971. Toutes les références en français sont (sauf mention contraire) issues de F. O'Connor, 2009.

2. «Madame, dit-il, la parole de dieu devrait être dans le salon » $(2009,314)$.

3. Ma traduction.

4. «Sois un homme. Ce n'est qu'un simple d'esprit que tu dois noyer " (ma traduction).

5. «Et puis, rester là, bouche ouverte comme un chimpanzé, devant une négresse qui t'renseigne... Grand Dieu ! ", (2009, 272).

6. «Mr Head, tassé comme un vieux singe sur le couvercle de la poubelle décide que si Nelson tardait à se réveiller, il ferait un grand bruit en donnant un bon coup de pied dans la poubelle » (2009, 272-273).

7. «Il imaginait sa voix hypnotique pénétrer jusqu'au garçon en passant par les profondeurs obscures de son psychisme " (ma traduction).

8. «Je ne sais pas, dit l'enfant, d'une voix boiteuse » (ma traduction).

9. « Ha mort, ha mort. Carn, carn, carn » (il est mort, il est mort. De la chair, de la chair, de la chair, Catalogne) ; « Coac ! coac ! coac ! vole de la carn! Vole de la carn! (couac ! couac ! couac ! Je veux de la chair ! je veux de la chair !, Auvergne). (Perbosc, 1988, 127).

10. «Il retint sa respiration comme s'il s'apprêtait à entendre une voix d'en haut. Au bout d'un instant, il entendit une poule qui grattait sous lui, sous la véranda » $(2009,377)$.

11. La tentative vaine de communication entre Bailey et la grand-mère est parallèle au langage de la basse-cour relevé et interprété dans le Quercy entre un poussin qui déplore la pluie et sa mère poule qui lui répond: «Piu! Piu ! Piu !/ Crac-t'aquela !» (Croque celle-là !) (Perbosc, 1988, 220).

12. "On a mangé avant de partir », $(2009,266)$, "T'as perdu le sac et la route. Tu peux bien attendre que j'me repose» $(2009,271)$.

13. «Aidez-moi à ne pas être aussi méchante, commença-t-elle mécaniquement, aidez-moi à ne pas empoisonner maman. Aidez-moi à ne pas parler comme je le fais » $(2009,257)$.

14. «Le vieillard se dit que si par hasard il voyait une bouche d'égout il tomberait dedans et se laisserait engloutir $(2009,275)$.

15. «Je sais que vous croyez à la charité chrétienne » $(2009,313)$.

16. «Je vois que vous vous êtes payé un joli petit saut!» $(2009,200)$.

17. "Mais ça aurait bien mieux valu pour tous que vous m'ayez pas repéré, ma petite dame » $(2009,200)$.

18. «J'suis très touché, madame » $(2009,201)$.

19. "Je vais me rendre compte tout à l'heure » $(2009,202)$.

20. «J'vais danser le rock and roll, le rock and roll. Pour sauver ma bon Dieu d'âme, j'peux pas contenter c'te femme. Sur quoi il décrivit de grands cercles en lançant latéralement sa jambe boiteuse. » $(2009,636)$.

21. «Jésus me sauvera, mais pas cet athée dégueulasse et menteur, pas ce... » (...), « Aux boiteux reviendra la proie », brailla-t-il, mais ses clameurs furent étouffées quand ils le poussèrent dans la voiture. » $(2009,659)$.

22. «Un chant populaire qui tenait de la romance et du cantique » $(2009,251)$.

23. Ma traduction.

24. «Ça doit être le taureau minable de quelque nègre » $(2009,533)$.

25. «Pourquoi tu ne le ramasses pas toi-même, papa?» $(2009,734)$.

26. Ma traduction. 
27. «Le bourrelet charnu de sa lèvre inférieure s'incurvait et, mieux qu'un écriteau, disait à tout un chacun : ‘ON EST PRIÉ DE NE PAS M'EMBÊTER' » $(2009,527)$.

\section{RÉSUMÉS}

Flannery O'Connor se donne pour mission de crier à l'oreille des sourds pour faire entendre la voix du mystère. C'est en faisant passer les personnages par la gueule du dragon (métaphore à choix multiples: Géorgie profonde, constellation, monstres à la voix déformée ou encore péripéties sonores du texte) que les nouvelles tentent de montrer la bonne voie. Les cris des galliformes (paons, poules et dindons) sont récurrents dans son œuvre et ce sont la matière et la manière de leurs voix qui éclairent le chemin. Les personnages ont un talon d'Achille vocal. Mais la voix est également le mystérieux véhicule de la révélation. Si les tonalités sont vicinales, elles sont transcendées pour être pleinement entendues dans les bruits, le rythme et les onomatopées. Tel un formidable don de voir, Flannery O'Connor souhaite partager sa connaissance d'une parole secrète, donner à entendre les voix des animaux, une sorte de langage mimologique connecté au divin.

Flannery O'Connor intends to shout at the deaf to make us hear the voice of mystery. Thus, her characters have to pass by the dragon (a multiple-choice metaphor: either you understand it as deep Georgia, a constellation, monsters with distorted voices or the sonorous adventures of the text). The galliformes's noises are recurrent in her work, notably those of peacocks, hens and turkeys: it is the matter and the manner of their voices that tell us the way to follow. The voice is something of an Achilles heel to most characters. It is also the voice that is the mysterious vehicle of revelation. If sounds are regional, they are transcended to be heard in noises, rhythms and onomatopoeias. As if O'Connor possessed the gift of (fore)sight, she wishes she could share her knowledge of a secret language, enable readers to listen to animal voices, a sort of mimological language connected to the divine.

\section{INDEX}

Mots-clés : voix vicinales, voix marginales, voix animales, bruits, O'Connor Flannery (1925-1964), paon, gueule du dragon

Keywords : Regional Voices, Marginal Voices, Animal Voices, Noises, O'Connor Flannery (1925-1964), Peacock, Dragon's Mouth

\section{AUTEUR}

\section{CLAUDIA DESBLACHES}

Université de Rennes 2 